Third Meeting, 14th January 1898.

J. B. Clark, Esq., M.A., F.R.S.E., President, in the Chair.

\title{
The Trisection of a Given Angle.
}

By Lawrence Crawford, M.A., B.Sc.

1. In a given circle let the arc AP subtend an angle $3 a$ at the centre $O$, it is required to trisect the angle $A O P$, or the arc AP.

The three trisectors will be $O Q_{1}, O Q_{2}, O Q_{3}$, where $A O Q_{1}=a$, $\mathrm{AOQ}_{2}=\frac{1}{3}(2 \pi+3 \alpha)=\alpha+2 \pi / 3, \quad \mathrm{AOQ}_{3}=\frac{1}{3}(4 \pi+3 \alpha)=\alpha+4 \pi / 3$

$\therefore Q_{1}, Q_{2}, Q_{3}$ form an equilateral triangle. (See Figs. 10 and 11.)

We proceed to solve the problem by drawing a conic through $Q_{1}, Q_{2}, Q_{3}$, and we wish to find in what cases such a conic can be drawn, a conic cutting the circle in four points, three of which form an equilateral triangle.

2. Take the circle as $x^{2}+y^{2}=d^{2}$, OA being the axis of $x$, and let the equation of the conic be

$$
a x^{2}+2 h x y+b y^{2}+2 g x+2 f y+c=0 .
$$

On eliminating $y$, the equation for the $x$ co-ordinate of a point of intersection is found to be

$$
\begin{array}{r}
x^{4}\left\{(a-b)^{2}+4 h^{2}\right\}+4 x^{3}\{g(a-b)+2 f h\}+2 x^{2}\left\{(a-b)\left(b d^{2}+c\right)+2 g^{2}\right. \\
\left.+2 f^{2}-2 h^{2} d^{2}\right\}+4 x\left\{g\left(b d^{2}+c\right)-2 f h d^{2}\right\}+\left(b d^{2}+c\right)^{2}-4 d^{2} f^{2}=0
\end{array}
$$

or, for brevity, $\mathrm{A} x^{4}+4 \mathrm{~B} x^{3}+2 \mathrm{C} x^{2}+4 \mathrm{D} x+\mathrm{E}=0$.

We can write down the sum of the four roots, the sum of their products two by two, the sum of their products three by three, and the product of the four in terms of these coefficients; but three of the roots, $x_{1}, x_{2}, x_{3}$, say, are $d \cos \alpha, d \cos (\alpha+2 \pi / 3), d \cos (\alpha+4 \pi / 3)$ $\therefore$ their sum is zero, the sum of their products two by two is $-3 d^{2} / 4$, and their product $1 / 4 d^{3} \cos 3 \alpha$, and the substitution of these values gives us, if $x_{4}$ be the fourth root,

$$
\begin{gathered}
\mathrm{A} x_{4}=-4 \mathrm{~B}, \quad-\mathrm{A} \cdot 3 d^{2} / 4=2 \mathrm{C}, \\
\mathrm{A} d^{3} \cos 3 \alpha / 4-\mathrm{A} x_{4} \cdot 3 d^{2} / 4=-4 \mathrm{D}, \quad \mathrm{A} d^{3} \cos 3 a \cdot x_{4} / 4=\mathrm{E},
\end{gathered}
$$

which, on eliminating $x_{4}$ give

$$
3 \mathrm{~A} d^{2}+8 \mathrm{C}=0, \quad \mathrm{~A} d^{3} \cos 3 \alpha+12 d^{2} \mathrm{~B}+16 \mathrm{D}=0
$$

and

$$
\mathrm{B} d^{3} \cos 3 a+\mathbf{E}=0 \text {. }
$$


Now if we had started by eliminating $x$ and obtaining a biquadratic in $y$, a similar procedure, on noting that three of the roots are $d \sin \alpha, \quad d \sin (\alpha+2 \pi / 3)$, and $d \sin (\alpha+4 \pi / 3)$,

would have given us the equations

$$
3 \mathrm{~A} d^{2}+8 \mathrm{C}^{\prime}=0, \quad-\mathrm{A} d^{3} \sin 3 a+12 d^{2} \mathrm{~B}^{\prime}+16 \mathrm{D}^{\prime}=0,
$$

and

$$
-\mathrm{B}^{\prime} d^{\prime} \sin 3 a+\mathrm{E}^{\prime}=0 \text {, }
$$

where $\mathbf{B}^{\prime}, \mathrm{C}^{\prime}, \mathrm{D}^{\prime}, \mathbf{E}^{\prime}$ are coefficients corresponding to $\mathrm{B}, \mathrm{C}, \mathrm{D}, \mathrm{E}$; the coefficient of the leading term in both biquadratics is the same.

3. These equations, (I.) and (II.), are the equations to be satisfied, we proceed to reduce them and to find which are independent. i.e.,

From the first equation of each set we see that $C, C^{\prime}$ are equal, $(a-b)\left(b d^{2}+c\right)+2 f^{2}+2 g^{2}-2 h^{2} d^{2}=-(a-b)\left(a d^{2}+c\right)+2 f^{2}+2 g^{2}-2 h^{2} d^{2}$

$$
\therefore \quad(a-b)\left(\overline{a+b} d^{2}+2 c\right)=0 .
$$

Now $(a-b)$ cannot be zero, as our conditions (I.) and (II.) hold wherever we take the axes, keeping the origin at $O$, but $(a-b)$ is not an invariant for such change of axes $\therefore$ cannot always be zero, $\therefore$ we have the condition

$$
(a+b) d^{2}+2 c=0 .
$$

With the help of this equation to eliminate $C$, the first equation of either (I.) or (II.) gives on simplification

$$
d^{2}\left\{(a-b)^{2}+4 h^{2}\right\}=16\left(f^{2}+g^{2}\right) .
$$

The other two equations of (I.) give now on reduction, using (1) and (2) where necessary,

$$
\left\{(a-b)^{2}+4 h^{2}\right\} d \cos 3 a=4\{2 f h-g(a-b)\} \quad .
$$

and

$$
\{2 f h+g(a-b)\} d \cos 3 a=h^{2} d^{2}-4 g^{2},
$$

but on multiplying crosswise, we find these are not independent, so we may omit the latter.

The simplification of the last two equations of (II.) gives two equations of a similar type, also not independent; the former of these is

$$
-\left\{(a-b)^{2}+4 h^{2}\right\} d \sin 3 a=4\{2 g h+f(a-b)\} .
$$

Now if we square equations (3) and (4) and add, we find after division by $(a-b)^{2}+4 h^{2}$ that we only get equation (2), hence equations (1), (3), and (4) are our only independent equations. 
From the last two we can solve for $f$ and $g$ in terms of $a, h$, and $b$, and using equation (1) for $c$, we have that the equation of the conic may be written

$$
\begin{array}{r}
2 a x^{2}+4 h x y+2 b y^{2}-d x\{2 h \sin 3 a+(a-b) \cos 3 a\}-d y\{-2 h \cos 3 a \\
+(a-b) \sin 3 a\}-(a+b) d^{2}=0 .
\end{array}
$$

4. From the equation $\mathrm{A} x_{4}=-4 \mathrm{~B}$, and the corresponding one, we get the coordinates $x_{4}, y_{4}$ of the fourth point of intersection,

$x_{4}\left\{(a-b)^{2}+4 h^{2}\right\}=d \cos 3 a\left\{(a-b)^{2}-4 h^{2}\right\}+4(a-b) h d \sin 3 \alpha$,

$y_{4}\left\{(\grave{a}-b)^{2}-4 h^{2}\right\}=-d \sin 3 a\left\{(a-b)^{2}-4 h^{2}\right\}+4(a-b) h d \cos 3 a$.

Note that for a conic with axes parallel to the coordinate axes, $h=0$ and the fourth point of intersection is $(d \cos 3 a,-d \sin 3 a)$, a fixed point for all such conics.

5. Take now some special examples. Suppose first the conic is a rectangular hyperbola, then $a+b=0, \therefore$ equation (1) gives that $c$ is zero, or that the conic goes through the origin: this, however, we know, for the origin is the orthocentre of the equilateral triangle $Q_{1} Q_{2} Q_{3}$ and a rectangular hyperbola circumscribing a triangle passes through its orthocentre.

The equation of the rectangular hyperbola with axes parallel to the coordinate axes reduces to

$$
x^{2}-y^{2}-d x \cos 3 \alpha-d y \sin 3 \alpha=0,
$$

so only one rectangular hyperbola with such axes can be drawn to solve our problem.

Its centre is $\left(\frac{1}{2} d \cos 3 a,-\frac{1}{2} d \sin 3 a\right)$, and its transverse axis is $d \sqrt{\cos 6 \alpha}$ parallel to the axis of $x$, if $\cos 6 \alpha$ be positive, but $d \sqrt{-\cos 6 \alpha}$ parallel to the axis of $y$, if $\cos 6 \alpha$ be negative.

Another simple solution can be got by making the rectangular hyperbola pass through $\mathbf{A}$ instead of putting $h$ zero.

The equation of the hyperbola is

$$
a\left(x^{2}-y^{2}\right)+2 h x y-d x(h \sin 3 a+a \cos 3 a)+d y(h \cos 3 a-a \sin 3 a)=0
$$

$\therefore \quad$ if it pass through the point $\mathrm{A},(d, 0)$ we find

$$
h=a \tan \frac{3 \alpha}{2} \text {, }
$$

and the equation reduces to

where $\lambda$ is $\tan \frac{3 \alpha}{2}$, and this is a unique conic. 
The centre of this conic is the point $\left(\frac{1}{2} d, 0\right) \therefore$ the radius $d$ of the circle is a diameter of the conic. Figure 10 is a rough drawing of the circle and the conic, $A$ being here the fourth point of intersection. This is the construction given in Taylor's "Geometry of Conics," Example 528.

6. Take now the conic as a hyperbola, eccentricity 2 , the condition for which is $(3 a+b)(a+3 b)=4 h^{2}$.

Draw a conic with axes parallel to the axes of coordinates, then $h$ is zero, take here $3 a+b$ zero, then the equation of the conic reduces to

$$
x^{2}-3 y^{2}-2 d x \cos 3 \alpha-2 d y \sin 3 a+d^{2}=0, \quad \text { a unique conic. }
$$

The centre of this conic is $\left(d \cos 3 \alpha,-\frac{1}{3} d \sin 3 \alpha\right)$, and transferred to parallel axes through this point, the equation of the conic is $x^{2}-3 y^{2}+\frac{4}{3} d^{2} \sin 3 \alpha=0$, so that the transverse axis is parallel to the axis of $y$ and is in length $4 / 3 d \sin 3 a$.

The fourth point of intersection is $(d \cos 3 a,-d \sin 3 a)$, so that the distance between it and the centre is the semi-transverse axis, hence it is a vertex.

Also, if $\mathrm{C}$ be the centre of the conic, $\mathrm{CP}$ is equal to the transverse axis, i.e., twice the semi-tranverse axis, but the eccentricity is 2 , so that $\mathbf{P}$ is a focus. The other vertex is the point $(d \cos \alpha, 1 / 3 d \sin 3 \alpha)$.

A rough figure is drawn (Fig. 11) to show this construction, which may be put as follows: Take $Q_{4}$ the end of the ordinate through $\mathrm{P}$ and trisect it in $\mathrm{V}$ and $\mathrm{C}$. With $\mathrm{C}$ as centre, $V$ and $Q_{4}$ as vertices, describe a hyperbola of eccentricity 2 , this will cut the circle in the points of trisection of the angle AOP. This is the construction given in Taylor's Conics, Example 390.

7. The conic which solves the problem may be a parabola or an ellipse, but these conics do not give such simple constructions as those two which have been drawn. 